\section{Metastatic extramammary paget disease, a remarkable clinical case and a brief review of a rare disease}

\author{
Leonor Fernandes, ${ }^{1}$ Joana Graça, ${ }^{1}$ \\ Leonor Vasconcelos de Matos, ${ }^{1}$ \\ Rita Sampaio, ${ }^{2}$ Mafalda Miranda \\ Baleiras, ${ }^{1}$ Filipa Ferreira, ${ }^{1}$ \\ Marta Mesquita Pinto, ${ }^{1}$ \\ Helena Miranda, ${ }^{1}$ Ana Martins ${ }^{1}$ \\ ${ }^{1}$ Medical Oncology Department, \\ Hospital São Francisco Xavier, Centro \\ Hospitalar Lisboa Ocidental; \\ ${ }^{2}$ Pathology Department, Centro \\ Hospitalar Lisboa Ocidental, Lisboa, \\ Portugal
}

\begin{abstract}
Extramammary Paget Disease (EMPD) is an often-misdiagnosed rare disorder, whose cause remains unknown. Diagnosis is confirmed by skin biopsy. Primary treatment for EMPD is surgery. Recurrence is common in the first two years and prognosis is good if the disease is localized and there is no underlying associated cancer. Patients with invasive and metastatic EMPD are uncommon and exhibit a poor prognosis, even when there is good response to a first chemotherapy line. Multiple chemotherapeutic regimens, with varying levels of success, have been attempted, but standard of care is not established. The central nervous system seems to be a common metastatic site with better survival than visceral metastasis. We report a case of metastatic EMPD that addresses the difficulties associated with the treatment of this rare disease, that has no current guidelines.
\end{abstract}

\section{Introduction}

Extramammary Paget Disease (EMPD) is an often-misdiagnosed rare disorder, that affects apocrine gland-rich sites, such as the anogenital area and axillae, and is characterized by a slow-growing, cutaneous adenocarcinoma that manifests as a chronic eczema-like rash. ${ }^{1-3}$ In western countries, women in the sixth decade of life seem to be the most commonly affected by EMPD, and vulvar Paget disease accounts for around $65 \%$ of EMPD. ${ }^{4,5}$

EMPD closely mimics other common cutaneous disorders and is often subjected to different empirical treatments before performance of skin biopsy, that ultimately gives the diagnosis. ${ }^{3}$ Presence of Paget cells, as well as typical Immunohistochemistry (IHC) staining, confirms EMPD. ${ }^{1}$ The histological differential diagnosis is with Bowen disease, melanocityc lesions, or rarely metastatic epidermotropic carcinomas. ${ }^{4}$

The neoplastic cells are positive for mucin, EMA, CEA, and low molecular weight cytokeratins (CK7, CAM5.2). In vulvar origin cases GCDFP-15 and androgen receptors are positive, and if perianal origin, CK20 is positive. The negativity for melan A or high molecular weight cytokeratins should help to distinguish EMPD from Melanoma or Bowen disease respectively. 3,4

EMPD mostly presents as an intraepithelial carcinoma but invasive disease can be present in up to $25 \%$ cases with the potential to evolve to metastatic disease. ${ }^{7-11}$ Early recurrence is common, in the first two years. ${ }^{3}$

There are no current guidelines for the primary treatment, or recurrence, of EMPD and Wide Local Excision (WLE) is accepted as the standard approach. ${ }^{12,13}$ Microscopic spread of disease with irregular borders or multi-focal lesions are common, making negative margins both difficult to obtain surgically and to ascertain pathologically. Although there are some conflicting findings in literature about the importance of negative margins, it seems that microsurgical techniques such as Mohs micrographic surgery show lower rates of recurrence and hence diminish the potential for invasive disease and distant metastasis. ${ }^{12,14}$

There are some therapeutic approaches, other than surgery, reported in literature, particularly in cases where WLE is not feasible as in some recurrences or advanced disease. These approaches - chemotherapy, radiotherapy as well as other more experimental techniques (laser ablation, photodynamic therapy) -, have varying levels of success and no stablished efficacy. ${ }^{12,14-16}$

Overall prognosis is good if the disease only affects the epidermal layer of skin, surgical margins remain free of disease and there is no underlying associated cancer. Several authors have shown that dermal invasion increases the risk of death by EMPD,${ }^{4}$ and the metastatic potential of this disease has only recently been recognized. Ohara et al. proposed the first TNM staging system for EMPD in 2016, although this was based on a retrospective study and in a Japanese population only and thus is considered provisory and is not widely used. ${ }^{4,17}$ Some studies suggest the use of sentinel LN biopsy to evaluate the prognosis of invasive EMPD, but most of them are small, and included patients with intraepithelial and
Correspondence: Leonor Fernandes, Centro Hospitalar de Lisboa Ocidental - Hospital São Francisco Xavier, Lisboa, Portugal

Tel.: +35 1964553711

E-mail: fernandes.leonor@gmail.com

Key Words: Extramammary Paget Disease; Vulvar disease; metastatic stage; brain metastasis.

Conflict of interest: the authors declare no potential conflict of interests.

Acknowledgments: We acknowledge the important contribution of the patients' willingness to collaborate through the conduction of her case. We acknowledge the contribution of Joana Tavares for her help in retrieving MRI images from this case. We acknowledge Francisca Costa for her help in reviewing language and content.

Received for publication: 13 August 2020. Accepted for publication: 20 September 2020.

This work is licensed under a Creative Commons Attribution-NonCommercial 4.0 International License (CC BY-NC 4.0).

COPyright: the Author(s), 2020

Licensee PAGEPress, Italy

Dermatology Reports 2020; 12:8841

doi:10.4081/dr.2020.8841

Lymph Node (LN) disease ${ }^{17-20}$.

Patients with metastatic EMPD exhibit a poor prognosis, with a 20 -fold increased risk of disease-specific death and a median Overall Survival (OS) of 1.5 years. ${ }^{21-23}$ Due to its rarity, advanced disease is only reported in small series and thus evidence baseddata on treatment approaches is lacking.

Multiple chemotherapeutic regimens for treatment of metastatic disease have been attempted, most commonly fluoropyrimidines based regimens (5-fluorouracil (5-FU) + cisplatin; 5-FU + epirubicin + carboplatin + vincristine + mitomycin $\mathrm{C}-$ FECOM; S1 monotherapy) or taxanes based regimens (docetaxel monotherapy; docetaxel + S-1; and cisplatin + epirubicin + paclitaxel - PET), each with varying levels of success. Taxanes therapy either combined or alone seems to be the best first-line agent, in particular, due to the easier use (regimens with 5-FU require continuous infusion for one-week and S1 therapy is not available in Europe). ${ }^{21}$ However, although in about half of the patients there is an initial response to treatment, they tend to evolve to disease progression. ${ }^{21,22}$

Recent investigations into the pathogenesis of metastatic EMPD have identified potential therapeutic targets. It seems that 
around $60 \%$ of EMPD have HER2 overexpression and thus some reports on the use of trastuzumab have yielded favorable responses. ${ }^{22}$ Proliferation and survival in EMPD seems to be related to activation and interaction of Paget cells with several signaling pathways as the RAS/RAF/MEK/ERK, the PI3K/AKT/ mTOR, the RANKL/RANK and others. Furthermore, it seems also that a considerable percentage of EMPD patients are mismatch repair deficient which suggests that immunotherapy can also be a therapeutic option. ${ }^{24}$

In the last years some authors have also suggested that the central nervous system is a common metastatic site and that it conceals better prognosis than visceral metastasis. ${ }^{11}$ To address this issue, we report a clinical case which puts in evidence the clinical practice challenges faced by clinicians due to the rarity of this disease and the lack of diagnosis and management guidelines.

\section{Case Report}

A 64-year-old Caucasian woman was diagnosed with a vulvar EMPD in 2008. At another institution, she underwent a partial vulvectomy, distal urethrectomy and two more re-excisions due to recurrence in the following years. The patient did not attend appointments after 2018 .

In June/2019, she was admitted to the emergency department after a seizure. The head Computed Tomography (CT) showed a left frontal lesion and a Magnetic Resonance Image (MRI) confirmed multiple brain lesions. A thoracic-abdominalpelvic CT did not identify other lesions and she underwent excision of the largest symptomatic left frontal brain lesion in the same month. The histopathology report revealed metastasis of a poor differentiated carcinoma, with a solid pattern and marked cytological atypia and the IHC was positive for CK7, CK8/18, CKAE1/AE3, GATA3, TTF1 (focal) e GDCFP15 (focal), and negative for CK20, napsin A, RE, melan A, p40 e S100.

The patient was discussed at multidisciplinary meeting and referred to Oncology and Radioncology Departments. In July/2019, she had no neurological symptoms or other complaints and a Positron Emission Tomography (PET) scan suggested malignancy in periaortic and pelvic lymph nodes and a lesion at the anterior wall of the vagina. At this point it was assumed a recurrent vulvar stage IV EMPD with cerebral, LN and loco-regional metastasis and she was proposed for hippocam- pal-sparing Whole-Brain Radiotherapy (WBRT) followed by systemic treatment with palliative intent.

She underwent WBRT in August/2019 (total dose of $27 \mathrm{~Gy} / \# 9$ ). Due to an urosepsis episode, she skipped the last session and the beginning of first line palliative chemotherapy had to be delayed. She started weekly Paclitaxel (TX) in September/2019, with good tolerance to treatment, reporting grade (G) $1 \mathrm{~b}$ fatigue and oral mucositis. After 12 cycles, there was evidence of LN disease response, but dubious response at brain level, as the MRI showed contrast uptake in a right cortical parietal area and disappearance of the other brain lesions previously described (Figure 1). After 20 cycles, she had a complete response which is still maintained after 10 months of WBRT plus starting TX, with only associated G1 fatigue and diarrhea. A chemotherapy holiday was proposed, but due to good tolerance and her willingness, she is scheduled for maintaining this regimen.

\section{Discussion}

Metastatic EMPD is an aggressive disease, with poor outcomes, without prospective studies available and with no standard effective systemic treatment. Additionally, many of the patients with this disease are elderly, which adds complexity to therapeutic choices. ${ }^{14}$

Caucasian women seem to be the most affected by EMPD and vulva is the com-
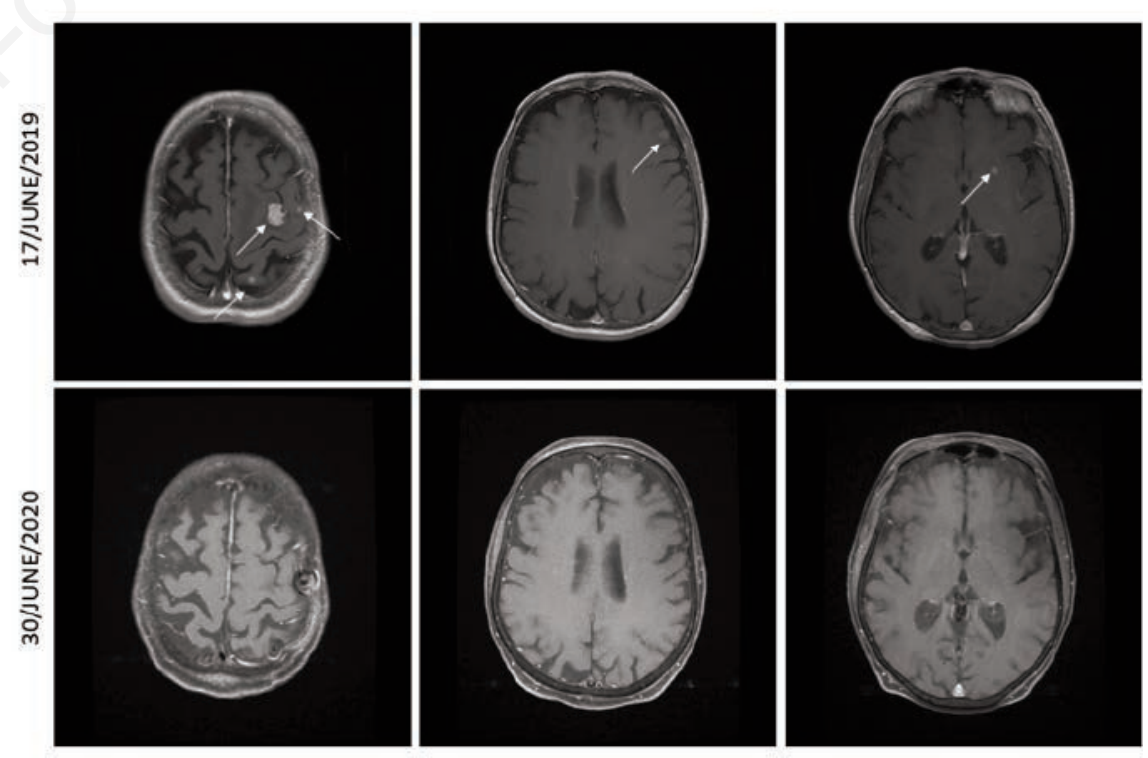

Figure 1. Evolution of MRI brain lesions from Case 2 the EMPD stage IV patient. Images from the line above are from brain MRI at diagnosis, and from the line below the latest, both sequences are T1 SE axial with gadolinium monest anatomical site. ${ }^{4}$

Standard treatment for EMPD is surgery, with a broad local resection, although differing approaches exist, depending on which specialty performs the surgical procedure. Wide resection of the local disease before lymphatic spreading seems to be the best strategy to prevent tumor-related death. ${ }^{14}$ When WLE is not possible, adjuvant radiotherapy (RT) can be proposed, although there is no consensus about how to adjuvant RT in similar cases, ${ }^{25}$ but Yao et al. ested that radiation could be deleridency could be related with radiation use more advanced disease.

Surgical excision is the first approach in recurrent EMPD but is frequently mutilating. Recently there are new treatment approaches with good results in disease control, like imiquimod 5\% cream and photoablation therapies, but our patient was not subjected to these experimental approaches. ${ }^{13}$

Although several factors have been associated with recurrence in in situ disease, information on markers for disease progression in the metastatic setting remain scarce. Over one-third of patients with LN disease subsequently develop distant metastasis. ${ }^{17}$ Tumor thickness, lymphovascular invasion, ber of LN metastases, vaginal EMPD, metastases, in particular visceral tors associated with poorer survival. ${ }^{4,26}$

Due to the rarity of this disease and in particular of stage IV patients, there is no manage these cases. Tolia et al. contemplate 
standard approach. ${ }^{11}$ Several mono or multi chemotherapeutic regimens have been used to treat metastatic EMPD; and despite initial response, few patients overcome tumor recurrence. ${ }^{22}$ Taxanes have been one of the most frequent drugs used in chemotherapy regimens for metastatic EMPD, in particularly docetaxel, with good results in terms of stabilization of the disease and favorable toxicity profile..$^{21,27}$

In this case WBRT and first line chemotherapy with paclitaxel succeed in achieving a complete response, which is rarely described in the literature, and the question now is whether this regimen should be maintained until progression or could be stopped and reintroduced after recurrence. In literature, first line chemotherapy seems to have good responses and brain metastasis have better prognosis than visceral disease, but OS at 5-years for stage IV EMPD is only $7 \%{ }^{17,22}$

For now, at our department, it was decided to maintain the current treatment as long as patient response, tolerance and acceptance are sustained.

\section{Conclusions}

With this case, we pretend to highlight the challenges faced by clinicians in treating metastatic EMPD and add some insights to current knowledge on this rare entity.

Chemotherapeutic regimens are only moderately effective, but there is growing evidence that taxanes are currently the best first line option in metastatic EMPD, although no prospective trial exists.

Current knowledge about metastatic EMPD suggests that in the future there will be options to therapeutic targets, that we hope can improve clinical outcomes. We also expect that further knowledge will allow EMPD management to be made with more certainty, given the actual lack of evidence and still incomplete understanding of this disease, in particular in the metastatic setting.

\section{References}

1. St Claire K, Hoover A, Ashack K, Khachemoune A. Extramammary Paget disease. Dermatol Online J 2019;25:112. Available from: https://escholarship.org/uc/item/7qg8g292\#article_abst ract

2. Merritt BG, Degesys CA, Brodland DG. Extramammary Paget Disease. Dermatol Clin 2019;37:261-7. doi:10.1016/j.det.2019.02.002
3. Zhao Y, Gong X, Li N, Zhu Q, Yu D, Jin $X$. Primary extramammary Paget's disease: a clinicopathological study of 28 cases. Int J Clin Exp Pathol 2019;12:3426-32. Available from: https://www.ncbi.nlm.nih.gov/pmc/articles/PMC6949851/

4. Morris CR, Hurst EA. Extramammary Paget Disease: A Review of the Literature-Part I: History, Epidemiology, Pathogenesis, Presenta-tion, Histopathology, and Diagnostic Workup. Dermatol Surg 2020;46:151-8.

5. Lopes Filho LL, Lopes IMRS, Lopes LRS, Enokihara MMSS, Michalany AO, Matsunaga N. Mammary and extramammary Paget's disease. An Bras Dermatol 2015;90:219-31.

6. Schmitt AR, Long BJ, Weaver AL, McGree ME, et al. Evidence-Based Screening Recommendations for Occult Cancers in the Setting of Newly Diagnosed Extramammary Paget Disease. Mayo Clin Proc 2018;93:87783. doi:10.1016/j.mayocp.2018.02.024

7. Jones ISC, Crandon A, Sanday K. Paget's disease of the vulva: Diagnosis and follow-up key to management; a retrospective study of 50 cases from Queensland. Gynecol Oncol 2011;122: 42-4.

8. Cai Y, Sheng W, Xiang L, Wu X, Yang H. Primary extramammary Paget's disease of the vulva: the clinicopathological features and treatment outcomes in a series of 43 patients. Gynecol Oncol 2013;129:412-6.

9. Fanning J, Lambert HC, Hale TM, Morris PC, Schuerch C. Paget's disease of the vulva: prevalence of associated vulvar adenocarcinoma, invasive Paget's disease, and recurrence after surgical excision. Am J Obstet Gynecol 1999;180:24-7.

10. Parker LP, Parker JR, Bodurka-Bevers D, Deavers M, et al. Paget's disease of the vulva: pathology, pattern of involvement, and prognosis. Gynecol Oncol 2000;77:183-9.

11. Fusumae T, Fukuda K, Hirai I, Nakamura Y, et al. Outcomes in extramammary Paget's disease patients with brain metastasis: a retrospective analysis. J Am Acad Dermatol 2020; S0190$\begin{array}{llllllllllll}9 & 6 & 2 & 2 & : & 3 & 0 & 9 & 6 & 6 & - & \mathrm{X}\end{array}$. doi:10.1016/j.jaad.2020.05.094

12. Long B, Schmitt AR, Weaver AL, McGree M, et al. A matter of margins: Surgical and pathologic risk factors for recurrence in extramammary Paget's disease. Gynecol Oncol 2017;147:35863. doi:10.1016/j.ygyno.2017.09.008

13. Edey KA, Allan E, Murdoch JB, Cooper S, Bryant A. Interventions for the treatment of Paget's disease of the vulva. Cochrane Database Syst Rev 2019;6: CD009245. doi:10.1002/ 14651858.CD009245.pub3

14. Simonds RM, Segal RJ, Sharma A. Extramammary Paget's disease: a review of the literature. Int J Dermatol 2019;58:871-9.

15. Cowan RA, Black DR, Hoang LN, Park $\mathrm{KJ}$, et al. A pilot study of topical imiquimod therapy for the treatment of recurrent extramammary Paget's disease. Gynecol Oncol 2016;142:139-43. doi:10.1016/j.ygyno.2016.04.028

16. Kilts TP, Long B, Glasgow AE, Bakkum-Gamez JN, Habermann EB, Cliby WA. Invasive vulvar extramammary Paget's disease in the United States. Gynecol Oncol. 2020; Available from: https://doi.org/10.1016/ j.ygyno.2020.03.018

17. Ohara K, Fujisawa Y, Yoshino K, Kiyohara Y, et al. A proposal for a TNM staging system for extramammary Paget disease: Retrospective analysis of 301 patients with invasive primary tumors. J Dermatol Sci 2016;83:234-9. doi:10.1016/j.jdermsci.2016.06.004

18. Nakamura Y, Fujisawa Y, Ishikawa M, Nakamura Y, et al. Usefulness of sentinel lymph node biopsy for extramammary Paget disease. Br J Dermatol 2012;167:954-6- doi:10.1111/j.13652133.2012.11017.x

19. Hatta N, Morita R, Yamada M, Echigo $\mathrm{T}$, et al. Sentinel lymph node biopsy in patients with extramammary Paget's disease. Dermatologic Surg 2004;30: 1329-34. doi: 10.1111/j.15244725.2004.30377.x.

20. Fujisawa Y, Yoshino K, Kiyohara Y, Kadono $\mathrm{T}$, et al. The role of sentinel lymph node biopsy in the management of invasive extramammary Paget's disease: Multi-center, retrospective study of 151 patients. J Dermatol Sci 2015;79:38-42.

21. Yoshino K, Fujisawa Y, Kiyohara Y, Kadono T, et al. Usefulness of docetaxel as first-line chemotherapy for metastatic extramammary Paget's disease. J Dermatol 2016;43:633-7.

22. Fukuda K, Funakoshi T. Metastatic extramammary Paget's disease: Pathogenesis and novel therapeutic approach. Front Oncol 2018;8:1-8.

23. Christensen S, Leffell D. Cancer of the Skin. In: DeVita, Hellman, and Rosenberg's Cancer: Principles \& Practice of Oncology. Philadelphia: Wolters Kluwer Health; 2015. p. 1333.

24. Kang Z, Xu F, Zhu Y, Fu P, et al. Genetic analysis of mismatch repair genes alterations in extramammary 
paget disease. Am J Surg Pathol 2016;40:1517-25.

25. Tolia M, Tsoukalas N, Sofoudis C, Giaginis C, et al. Primary extramammary invasive Paget's vulvar disease: What is the standard, what are the challenges and what is the future for radio- therapy? BMC Cancer 2016;16:10-7. 27. Nakamura Y, Tanese K, Hirai I, Fukuda doi:10.1186/s12885-016-2622-5

26. Yao H, Xie M, Fu S, Guo J, et al. Survival analysis of patients with invasive extramammary Paget disease: Implications of anatomic sites. BMC Cancer 2018;18:1-9.
$\mathrm{K}$, et al. Weekly docetaxel monotherapy for metastatic extramammary Paget's disease: Retrospective single-institute analysis. J Dermatol 2020;47:418-22. 